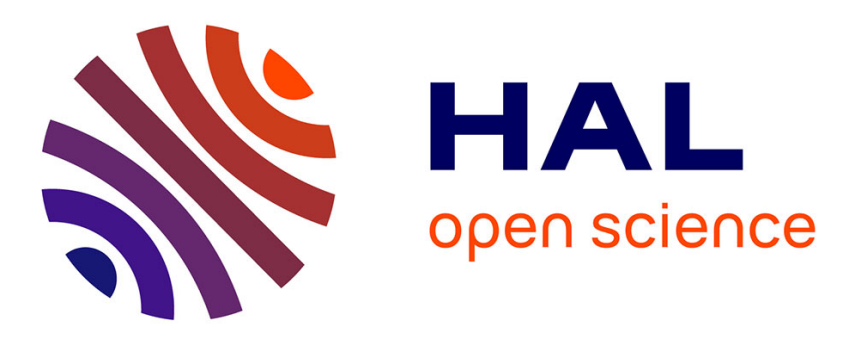

\title{
Analyse lexicale des sources d'influence dans les jurys d'assises.
}

Patrick Scharnitzky, Nikos Kalampalikis

\section{To cite this version:}

Patrick Scharnitzky, Nikos Kalampalikis. Analyse lexicale des sources d'influence dans les jurys d'assises.. Bulletin de psychologie, 2007, 60 ((5)), pp.425-432. halshs-00533470

\section{HAL Id: halshs-00533470 \\ https://shs.hal.science/halshs-00533470}

Submitted on 28 Dec 2018

HAL is a multi-disciplinary open access archive for the deposit and dissemination of scientific research documents, whether they are published or not. The documents may come from teaching and research institutions in France or abroad, or from public or private research centers.
L'archive ouverte pluridisciplinaire HAL, est destinée au dépôt et à la diffusion de documents scientifiques de niveau recherche, publiés ou non, émanant des établissements d'enseignement et de recherche français ou étrangers, des laboratoires publics ou privés. 


\section{Analyse lexicale des sources d'influence dans les jurys d'assises \\ SCHARNITZKY Patrick* KALAMPALIKIS Nikos**}

Un des axes majeurs de la recherche sur la justice en psychologie sociale porte sur l'usage de notions intuitives, fragments de pensée quotidienne, intervenant dans la construction, l'élaboration et le rendu d'un jugement en situation de groupe. Un des terrains de prédilection d'observation et d'investigation systématiques de cette «justice du sens commun » est celui des jurys populaires (voir Lieberman, Sales, 1997 ; Finkel, 1995). Des études en psychologie sociale appliquée à la justice (voir Rainis, 2002 ; Guingouain, Manchec, Testé, 2002 Scharnitzky, 2006) font la différence entre les variables légales (celles qui sont légitimes dans la prise de décision et sur lesquelles les jurés sont officiellement invités à fonder leur intime conviction) et les variables extra-légales (celles qui ne sont pas légitimes et dont on demande explicitement aux jurés de ne pas tenir compte dans leur opinion). Dans la première catégorie, on retrouve des variables telles que les auditions, les témoignages, les plaidoiries, mais aussi et, surtout, le type de crime commis et la gravité du préjudice résultant du crime. Dans la seconde catégorie, on peut citer, par exemple, les caractéristiques sociodémographiques de l'accusé et de la victime ou encore la durée du procès et sa médiatisation. Si on peut logiquement concevoir l'influence que peuvent représenter les variables légales sur la prise de décision, il est, en revanche, plus difficile d'envisager que les jurés soient aussi soumis à des sources d'influence « illégitimes ».

Une étude récente (Scharnitzky, Rainis, 2006 ; Richard, Finkelstein, Oberlé, Rainis, Scharnitzky, 2008) par questionnaire, sur un échantillon représentatif de 700 citoyens français, potentiellement jurés, a exploré différents aspects de la représentation sociale de la fonction de juré d'assises. Outre la méconnaissance de la justice et la méfiance quant à son fonctionnement et à son efficacité, cette enquête a exploré la question de l'impact de ces variables légales et extra-légales sur la prise de décision. Il faut préciser qu'il s'agissait d'opinions sur la façon dont les personnes interrogées pensaient qu'elles réagiraient si elles étaient désignées comme jurés. Il leur était proposé une liste de variables, légales et extra-légales (sans qu'elles soient, bien entendu, classées et nommées comme telles) et il leur était demandé de dire, dans quelle mesure, d'une part, ils pensaient que ces variables pourraient les influencer personnellement et, d'autre part, comment elles pourraient influencer les jurés, en général. L'objectif était de mesurer un décalage possible entre eux-mêmes et les autres. Les variables légales furent significativement perçues comme les plus influentes et ne firent apparaître aucune différence entre l'influence exercée sur soi et celle exercée sur les autres. En revanche, si les variables extra-légales étaient jugées avoir moins d'influence, les personnes interrogées exprimaient, de façon systématique, qu'elles en auraient davantage pour les autres que pour euxmêmes. Ils disaient qu'ils seraient moins influencés que les autres par la personnalité du président de la cour d'assises, par le comportement des autres jurés, lors des délibérations et par la médiatisation de l'affaire. On comprend, ici, que les jurés potentiels manifestent une forme de défense contre des sources d'influence, qu'ils savent illégitimes, en construisant l'illusion qu'ils y résisteraient mieux que les autres.

Nous avons souhaité croiser ces résultats avec une étude portant sur une population qui a réellement vécu l'expérience d'être juré d'assises, en réalisant une série d'entretiens semi-directifs, explorant différents aspects de ce vécu, dont la question des influences subies. Cet article se centre sur les trois variables extra-légales, qui peuvent devenir des sources d'influence importantes, et qui nous semblent les plus saillantes, au regard de l'étude par questionnaire que nous venons de citer, à savoir la personnalité et le rôle joué par le président de la cour, l'impact de la dynamique de groupe dans la prise de décision collective, en vue du rendu du verdict, et le rôle joué par les médias dans cette situation de jugement. Si des jurés potentiels se disent capables de

\footnotetext{
* Université de Picardie Jules-Verne, Département de psychologie, Chemin du Thil, 80000 Amiens. <patrick.scharnitzky@u-picardie.fr>

** Université Lyon 2, Institut de psychologie, Groupe de recherche en psychologie sociale (EA GRePS), 5 avenue P. Mendès-France, 69676 Bron cedex.
} 
résister à de tels facteurs, qu'en est-il de la part de citoyens qui ont concrètement rempli ce rôle ? Notre hypothèse est la suivante: trois variables extralégales (le comportement du président de la cour, la dynamique de groupe, lors des délibérations, le rôle des médias), représentent des sources de l'influence subie par les jurés d'assises, repérables dans le discours relatant leur vécu du procès.

\section{ÉCHANTILLON, CORPUS, MÉTHODE}

Notre échantillon est composé de quarante anciens jurés (vingt hommes, vingt femmes) contactés grâce à l'aide du tribunal de grande instance d'une grande région française (pour une description du fonctionnement d'une cour d'assises, voir annexe 1). Tous ont été jurés ces quatre dernières années. Du point de vue sociodémographique, leur âge moyen est de 47,6 ans, ils ont en moyenne un enfant, treize d'entre eux mènent une vie maritale. Les sujets ont été interrogés individuellement dans un entretien semi-directif, dont le guide a été structuré essentiellement autour des trois temps de cette expérience (avant, pendant, après le procès). Notre corpus a donc été constitué à partir des retranscriptions des quarante entretiens. Notons, d'emblée, sa taille particulièrement importante (environ 500000 mots). Cet important volume lexical peut s'expliquer, aussi bien par la méthode d'interrogation - ici l'entretien-, permettant un approfon- dissement du discours, grâce à l'interaction, constituant ainsi « un événement de communication intersubjective » (Kalampalikis, Moscovici 2005, p. 16), que par la volonté de tous les anciens jurés d'exprimer dans le détail et de faire partager le vécu de leur expérience. Ajoutons à cela la variable « date » du procès, assez proche de l'entretien, optimisant la remémoration des événements. Pour l'analyse de ce corpus imposant, nous avons utilisé une analyse informatique du discours, sémantique et lexicométrique, grâce au logiciel Alceste (Reinert, 2003). Naturellement, il n'est pas question, dans le cadre de cet article, de présenter le détail de tout le corpus analysé (Scharnitzky, Kalampalikis, Durif-Varembont, Mercader, 2006). Nous nous centrons uniquement sur l'identification des espaces de références des différentes formes d'influence rapportées dans le discours de jurés, afin de mesurer leur nature, leur impact et la façon dont elles ont été vécues et verbalisées.

\section{RÉSULTATS}

Notre démarche a consisté, dans un premier temps, à localiser les lieux de discours, où les sources d'influence étaient les plus présentes et les plus pertinentes. L'analyse lexicale, par la méthode Alceste ${ }^{1}$, nous a permis d'identifier deux pôles majeurs de références, représentés par le graphique 1 , issu de la classification descendante hiérarchique.

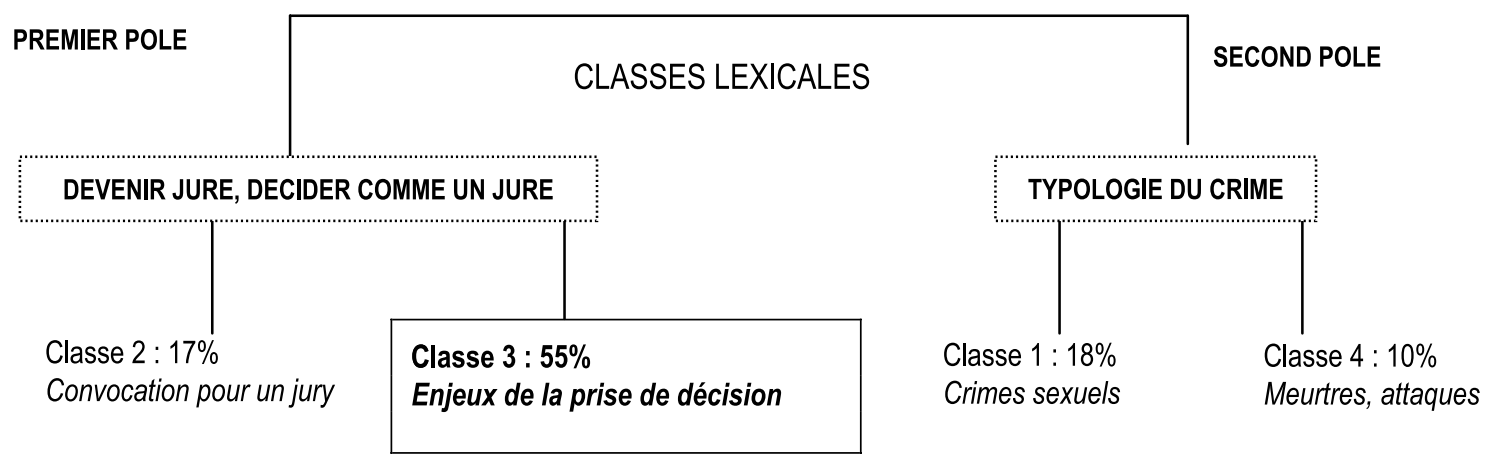

Graphique 1. Aperçu des classes lexicales issues de l'analyse Alceste.

Notons, d'emblée, que le graphique 1 incorpore un début d'interprétation, corroboré par les différents indices statistiques et lexicaux de l'analyse (profil des classes, vocabulaire spécifique, analyse factorielle des correspondances, segments répétés, etc.). Le premier pôle (classes 2 et 3 ), regroupe les souvenirs de la convocation à participer à un jury d'assises, l'expérience de cette participation et, notamment, les enjeux psychosociaux en œuvre dans le processus d'une prise de décision collective. Le second pôle (classes 1 et 4), porte sur la nature des affaires jugées, avec une

1. Rappelons, ici, brièvement, les principes de la méthode Alceste : elle repose sur un découpage du corpus déclinaison nette entre, d'une part, majoritairement, les crimes de viol et, d'autre part, principalement, les meurtres (voir Mercader et coll., dans ce dossier).

C'est surtout au niveau du premier pôle et, tout particulièrement, de la classe 3 , que notre attention

en fragments de taille relativement analogues, dits « unités de contexte ». Ces mêmes fragments sont ensuite classés statistiquement selon une procédure descendante hiérarchique, dont l'objectif est la répartition des énoncés en classes, marquées par le contraste de leur vocabulaire. Cette méthode permet, donc, de mettre en lumière les traces lexicales les plus prégnantes de ces espaces de référence, les «mondes lexicaux » (voir Kalampalikis, 2003 ; Kalampalikis, Moscovici, 2005 ; Reinert, 2003). 
s'est portée. Elle représente le champ lexical le plus investi de la part de nos sujets (55\% de l'ensemble) et correspond au véritable vécu de leur expérience, le vocabulaire spécifique ci-dessous ${ }^{2}$ (tableau 1 ) en témoigne largement :

presid+ent (117.70), deliberat+ion (97.61), peine+ (94.45), groupe+ (77.18), opinion+ (72.55), chang+er (72.01), justic< (70.65), pos+er (69.22), note+ (59.25), question+ (58.79), vote+ (54.33), role+ (50.88), vous (50.81), avocat+ (45.93), influenc+er (45.81), gens (44.05), professionn+el (43.10), on (42.07), import+ant (41.54), assesseur+ (38.28), percevoir (38.15), trop (37.10), parole+ (36.79), conviction+ (35.53), point de vue+ (32.37), experience+ (32.02), discut+er (31.25), dire+ (27.81), conscienc+e (27.30), trouv+er (26.93), avis (26.69), dout+e (26.55), finalement (26.50), seance+ (25.29), decis+ion (24.60), vot+er (24.58), ecout+er (24.32), reflex+ion (23.82), mesur+er (23.66), temps (23.38), citoyennete (22.15), evoluti+f (22.02), pens+er (21.68), moi (20.18), societe+ (20.01), intim+e (20.00), falloir (19.72), je (17.09).

Tableau 1. Vocabulaire spécifique de la classe 3 (par $\chi^{2}$ décroissant)

La notion $\mathrm{du}$ « groupe », apparaît, ici, très fortement $(91,4 \%$ de présence exclusive) liée, aussi bien au dispositif de la séance qu'au processus de décision, tel qu'il se construit dans et par le déroulement du procès. Pour preuve, l'emploi significatif de verbes relatifs à ce processus (écouter, changer, penser, mesurer, trouver, voter, poser des questions, exprimer, influencer, manipuler, décider, délibérer, etc.), illustrant la part intentionnelle de l'individuel dans la prise collective d'une décision d'importance. Il est essentiel de noter que le discours, ici, ne s'exemplifie pas par rapport à des cas concrets d'affaires jugées, mais reste sur le plan de la description de l'évolution d'une situation, d'un procès X ou Y. Certains constats lexicaux et quantitatifs supplémentaires nous permettent d'identifier d'autres spécificités du lexique composant ce champ lexical, grâce, notamment, aux mots, qui apparaissent exclusivement ou majoritairement dans ce dernier. Par exemple, $78 \%$ des occurrences totales du terme «président-e » se retrouvent dans cette classe, ainsi que 72,6\% du mot « avocat(s) », $97,6 \%$ des occurrences du mot « délibération(s) », $94 \%$ du mot « vote », 92,8\% du verbe « voter», 94,5\% du mot « peine », $100 \%$ du mot « médias » et $100 \%$ du mot « journaliste(s) »; enfin, $87 \%$ des occurrences du mot «influence(s) » et $80,6 \%$ du mot « doute ».

La place et l'impact des acteurs institutionnels du procès (le président de la cour d'assises, les avocats), la délibération collective, lors du rendu du verdict, le rôle des médias, voici trois pistes, suggérées directement par le lexique de ce champ, qui corroborent notre problématique du point de vue de la significativité de la présence de variables extra-légales dans le discours des anciens jurés. Par la suite, nous allons entrer dans le détail de chacune de ces trois variables. Cependant, précisons, d'emblée, que nous avons choisi d'illustrer le contenu de chacune d'entre elles par des extraits. Ces derniers tirent leur légitimité statistique de la significativité des entretiens, dont ils sont tirés, dans la construction de la classe en question
(«Enjeux de la prise de décision ») qui, rappelons-le, occupe $55 \%$ de l'ensemble du corpus.

\section{Le président de la cour}

Nos interviewés évoquent, sans exception, l'impact de la présence et de la participation du président de la cour, tant au niveau de la procédure que de la délibération. Le mot « Président » est cité 503 fois au total par l'ensemble des sujets dont 392 fois $(78 \%$ ), dans la classe étudiée. En outre, ce même mot (avec un $\chi^{2}$ de 117,7) est le plus fréquemment cité. En effet, le président de la cour se pose à la fois en gestionnaire des débats et en porteur d'un savoir «objectif», source indéniable d'autorité. Toutes les conditions sont, donc, réunies pour mettre les jurés en situation d'influence, volontaire ou involontaire, à cette forme d'autorité judiciaire. Dans l'argumentation des anciens jurés, les différents degrés de l'influence perçue de cette source d'autorité varient, allant du poids de la présence à la pression, de l'incitation à la suggestion, de l'influence à la manipulation, dont les quelques extraits ci-dessous témoignent largement :

- «Bon la présidente du tribunal et ses deux assesseurs en fait ont déjà un petit peu une idée de l'affaire qui va être débattue en fait, jugée, et ils influencent beaucoup, donc quand on avait une petite pause, par exemple la présidente venait nous voir, ou ses assesseurs, chacun son tour avait toujours un mot dans un sens où il voulait vraiment qu'on aille en fait. » [N10/Homme/2001] ${ }^{3}$.

- «Par rapport à cette affaire-là, le président a été très très étonné, qu'on ait mis que cinq ans. Et par contre quand il a fallu mettre du sursis, il a fait un forcing pour qu'on ne mette pas de sursis. » [N7/Homme/2003].

2. Le signe « + » résulte d'une opération standardisée de lemmatisation réalisée par le logiciel Alceste, consistant à réduire les différentes formes lexicales à leur racine (lemme), à l'aide de dictionnaires de reconnaissance modulables.

3. Codification: $\mathrm{N}^{\mathrm{o}}$ d'entretien $(\mathrm{N})$, sexe, date du procès. 
- «Quelque part, je me suis dit, alors je ne sais pas quel est le rôle du président quand il est avec les jurés. Je ne sais pas si c'est un rôle d'orientation, de canaliser les choses mais je pense que quelque part il a influencé... il a influencé quand même les jurés. » [N36/Femme/2002].

- «Vous subissez trop de choses par exemple la pression d'un magistrat, et quand je dis pression, c'est une pression tout en douceur tout en sourire hein. » [N37/Femme/2002].

- «Enfin, je suppose que c'est pareil pour les autres présidents de cours d'assises, mais elle avait beaucoup de poids sur nos discussions. » [N8/Femme/2001].

- « Je compare toujours un petit peu quand les gens veulent nous mettre une nouvelle version dans le travail et qu'il faut absolument qu'on ait cette nouvelle version, et puis qu'ils viennent et puis qu'ils nous réunissent en nous disant on va vous demander votre avis alors que l'avis est déjà fait et refait... Un peu moins ici, car quand même on vote, on ne peut pas faire autrement, mais c'est quand même bien dirigé quoi, donc voilà. » [N5/Femme/2003].

Dans une moindre mesure, on peut aussi évoquer le rôle joué par les avocats et les experts, qui sont aussi porteurs d'une certaine forme de crédibilité, $\mathrm{du}$ fait de leur statut respectif, mais qui est amoindrie par les intérêts unilatéraux perçus dans les propos de chacun. De plus, la décision du juré n'étant pas individuelle, ce dernier peut trouver une forme de dilution de sa responsabilité dans le fait que la décision incombe au groupe et non à sa seule intention personnelle.

\section{La délibération}

Au moment du rendu du verdict, la délibération prend forme dans une dynamique de groupe, dont les mécanismes d'influence sont bien connus (voir Moscovici, Doise, 1992 ; Mugny, Oberlé, Beauvois, 1995). Si on reprend la liste du vocabulaire spécifique de la classe 3 , nous constatons que trois des cinq mots ayant les $\chi^{2}$ les plus élevés font directement référence à la phase de délibération. Il s'agit des mots «délibération » $\left(\chi^{2}=97,6\right)$, « groupe » $(77,2)$ et l'addition des mots « vote » et « voter» $(79,9)$. Le mot «délibération » est cité 277 fois au total, dont 232 fois exclusivement dans la classe « enjeux de la prise de décision ». Ici, le groupe intervient aussi bien comme cadre structurant, révélateur d'appartenances sociodémographiques (par exemple, le sexe, la catégorie socioprofessionnelle) devenant facteurs d'homogénéité ou d'hétérogénéité, que comme cadre dynamique, au sein duquel les avis, les opinions, les appréciations personnelles, peuvent et doivent évoluer :

- «C'est difficile, je ne sais pas, on est quand même pris dans un phénomène de groupe hein, oui, on a notre opinion, on peut l'exprimer, mais en même temps je pense qu'on ne veut pas trop se démarquer du groupe, je pense qu'il y a ça qui joue. » [N23/Femme/2003].

- «Quand je dis, je porte un jugement en mon âme et conscience, je suis chargé de plein de préjugés, je suis un produit de la société, donc mon «âme et conscience », je me suis représenté une composante de la société, je juge avec ça, j'suis calibré, comme j'ai vu que tous les autres jurés étaient calibrés. » [N29/Homme/2001].

- «Je crois qu'y avait une majorité d'hommes quand même, et y avait je crois, deux femmes, c'est tout, et ces femmes ouais aussi en tant que juré, un comportement je trouve très bizarre aussi pour être des femmes en plus alors que, bon, les hommes nous... » [N31/Homme/2001].

- «Il a pris douze ans, douze ans !, alors que moi c'est vrai que, personnellement j'ai été très déçue qu'il prenne que douze ans on a été pas mal, ouais, en fait, c'est simple, c'est bête, mais les femmes, on voulait au moins quinze ans, et les hommes moins, alors pourquoi je, parce que c'était peut-être un homme moi j'en sais rien.» [N2/Femme/2002].

- « Justement, j'ai trouvé que ça avait super bien évolué, je me suis dit les gens ils ont vachement vite changé d'avis. Parce qu'on a dû délibérer durant $2 \mathrm{~h}-2 \mathrm{~h} \mathrm{30}$, pour les deux c'était à peu près pareil. Mais il y avait quelqu'un qui avait marqué pour la deuxième affaire vingt cinq ans, c'était énorme, mais après du coup c'est tombé tout de suite. Parce que bon on fait un tour, on dit tant de dix ans, tant de trois ans, tant de cinq ans... C'est pour ça que parfois ça dure, ça dure. Parce que si les gens ne changent pas d'avis. On enlève un an par tour quand on n'est pas d'accord. Par exemple si le dernier il est quinze ans, on ne peut pas donner au-delà de quatorze, donc on enlève, donc ça peut durer... Et là j'ai trouvé que c'était vraiment vite, parce que les gens sont arrivés quand même à changer assez vite. » [N5/Femme/2003].

- «Écoutez sur quatre fois on était trois fois très homogènes, pas du tout de la même catégorie socio-professionnelle d'accord, pas homogène à ce point-là, mais homogène, sur le plan de la décision qu'on allait prendre, on a été, à chaque fois très rapide, y a qu'une fois où j'vous dis ça a été un peu long, parce qu'on était moins homogène, comme groupe, et parce que c'est vrai que le contexte à huis clos avait impressionné certains. » [N13/Femme/2002].

\section{L'impact des médias}

Le procès aux assises est une procédure pénale, qui s'inscrit dans une culture, dans un système politique et dans un cadre législatif, façonnés, de plus 
en plus, par la communication de masse. Dans ce sens, il est impossible d'occulter la dimension médiatique dans la prise de décision (Lombard, 1993 ; Beauvallet, Cirendini, 2004), même si elle occupe une place moindre que celle des deux autres sources d'influence précédemment exposées. Néanmoins, il est frappant de constater que $70 \%$ des jurés interrogés évoquent des références cinématographiques et exclusivement en lien avec la prise de décision. De plus, les mots «médias » et «journaliste(s) » sont cités 73 fois et, toujours, à l'intérieur de la classe analysée. Cet appel narratif à un réservoir culturel commun, cette traduction de l'expérience vécue par la mobilisation de lieux d'ancrage (voir Jodelet, 2006) - exposé dans le cas de la loi par Amsterdam et Bruner (2000) - leur sert à triple titre :

$1^{\circ}$ comme seul point de repère du fait juridique préalable au procès :

- « Là on se dit on n'est pas dans un film on est dans la réalité et on met derrière des choses assez concrètes derrière tout ce qu'on voit, dans les médias, on refait le lien. » [N29/Homme/2001].

- «Pour moi c'était une découverte c'est-à-dire bon je ne savais pas du tout comment ça se passait excepté que j'avais vu le film avec Henri Fonda. » [N9/Homme/2000].

- « Les gens, enfin on a tous vu des procès à la télé euh. En film au moins je crois. Donc c'était cette représentation que j'avais là sur la forme. Mais sur le fond (...). Sur son fonctionnement, si ce n'est ce qu'on en voit à la télé. Bien souvent c'est du cinéma donc c'est des films américains ou à part Lelouche je crois où ça se passe longtemps enfin bref, je n'avais pas d'idée sur le fonctionnement en lui-même. » [N11/Homme/2000].

$2^{\circ}$ comme analogie, pour illustrer, verbaliser, faire comprendre et transmettre leur récit :

- «Mais cela me faisait penser un petit peu à des plaidoiries que l'on voit parfois dans les films ou des choses comme ça de l'extérieur, mais il était génial il m'a époustouflé dans sa prestation. » [N3/Homme/2003].

- « Il mentait là aussi, et après y'avait toute une histoire, un véritable film, avec des dialogues d'Audiard. » [N23/Femme/2003].

- «C'est vrai que c'est impressionnant, et puis quand $\mathrm{y}$ faut se lever, et dire jurer machin c'est vrai que, ça fait quelque chose quoi c'est, c'est bizarre on se sent à la fois, irréel et à la fois c'est très concret quoi, c'est comme moi la première fois moi j'avais jamais vu une, une salle de tribunal et, c'est vrai avec toutes ces tentures rouges un peu à l'ancienne, surtout à Lyon là, c'est vrai que ça fait bizarre quoi, on se sent un peu dans un film, dans un décor. » [N34/Femme/2001].
Enfin, $3^{\circ}$ comme contre-exemple, pour parler d'une « autre » réalité, « moins vraie » que celle du procès, auquel ils ont assisté :

- «Moi ça m'a fait bizarre parce que je me suis dit : c'est réel, c'est vraiment réel, ce n'est pas du blabla. Ce n'est pas un film, ce n'est pas du cinéma (...), malheureusement c'est vrai. » [N28/Femme/ 2003].

- «Je ne suis pas dans un film, mais je vis un truc que des gens ont vécu avec tous leurs états d'âme. » [N19/Homme/2001].

- «On découvre un autre monde je dirais, enfin ça fait partie de notre monde mais on s'aperçoit rapidement que les faits divers qu' on peut lire occasionnellement sur les journaux ne sont pas, c'est un peu plus réel on va dire, plus proche de ce qu'on peut voir dans certains films. » [N6/Femme/2002].

\section{DISCUSSION}

Dans le cadre de cet article, nous avons cherché à illustrer l'apport de certaines théorisations, issues de la psychologie sociale (l'influence sociale, la pensée sociale), dans l'analyse du fait juridique, à partir du vécu d'un échantillon de citoyens, qui ont rempli le rôle de juré dans des procès aux assises. Cette expérience, capitale pour leur vie, a une portée politique et sociétale importante. Aux antipodes d'un processus de traitement individuel et rationnel de l'information, la situation du procès implique l'émotion, l'affect, l'interaction, la dynamique du groupe.

Concernant la première variable extra-légale, à laquelle nous nous sommes intéressés, le président de la cour, il est clair que cette influence est avérée dans le discours des jurés, comme les quelques extraits cités dans les résultats l'illustrent. Nous pouvons ici faire aisément référence aux travaux classiques de Milgram (1974), qui démontrent que, quand un contexte social réunit toutes les conditions favorables à un acte d'obéissance, l'individu est susceptible de se soumettre à une autorité sous trois conditions : il faut que l'autorité soit perçue comme légitime, cohésive et déresponsabilisante. Le président de la cour remplit, de fait, les deux premières, car il est légitime par son statut et son expertise, et forcement cohésif, puisqu'il est le seul à remplir ce rôle. En ce qui concerne la troisième, il faut souligner que les trois jurés professionnels (le président et ses deux assesseurs) prennent part au vote. On peut, donc, penser que les jurés peuvent confortablement « se ranger » à l'opinion du président, en considérant qu'il est suffisamment expert pour porter une partie, au moins, de la responsabilité du verdict.

Concernant la seconde variable, centrée sur l'impact de la délibération, les résultats mettent en 
évidence, là encore, une forte influence subie et verbalisée de la part des jurés interrogés. On voit bien, dans les extraits, que même si cette dernière existe, elle n'est pas toujours de même nature. Pour expliquer cette variabilité, on peut se référer aux deux processus «opposés », que sont la normalisation et la polarisation collective. La normalisation s'observe quand les acteurs n'ont pas d'idée $a$ priori sur la norme existante, dans le contexte social du jugement (voir Sherif, 1947 ; Doise, Mugny, 1995). Dans le cas qui nous intéresse ici, le jury devrait construire une réponse commune convergente, donc, modérée, à l'intersection des opinions individuelles. La mise en groupe aurait, alors, l'effet de modérer les opinions les plus radicales, au profit d'un consensus moyen. De plus, chaque juré est là pour la première fois (et, souvent, la dernière), ce qui lui retire toute capacité à s'appuyer sur une norme extérieure, tant la situation est exceptionnelle.

À l'inverse, la polarisation collective correspond à une radicalisation des opinions individuelles, par le simple jeu de la discussion collective (voir Moscovici, 1992). Pour ce faire, la discussion doit respecter deux conditions : les acteurs doivent se sentir fortement impliqués dans la discussion et un désaccord «minimum » entre des opinions, allant globalement dans le même sens, doit exister. Ainsi, l'échange de nouveaux arguments, le jeu de l'engagement social des acteurs et le poids des positions extrêmes poussent le groupe à formuler une opinion plus radicale que ne le serait la moyenne des opinions individuelles, avant le début de la discussion. Là encore, on peut imaginer un tel scénario pour une délibération de jury d'assises, car l'implication est naturellement forte de la part des jurés et les désaccords, quant à la durée de la peine, essentiellement, sont classiques.

Normalisation ou polarisation collective, les deux phénomènes sont envisageables, lors des délibérations de jury d'assises. Si le cas est univoque et le groupe assez homogène, on fera l'hypothèse d'une normalisation de la décision de groupe, qui se traduira par un verdict consensuel et « moyen ». Si l'accusé avoue sans hésitation, si les faits sont accablants pour lui et/ou si les preuves sont manifestes, le verdict est le fruit d'un consensus non conflictuel. En revanche, si les faits sont opaques, et si les témoignages et les expertises sont contradictoires, les jurés peuvent se trouver aisément en désaccord sur le degré de culpabilité ou de préméditation, par exemple, ce qui peut induire un phénomène de polarisation collective, radicalisant potentiellement la nature du verdict, à cause de l'aspect collectif de la décision. Selon un tel schéma, le jury devrait proposer une réponse collective plus extrême que ne le serait la moyenne arithmétique de leurs opinions individuelles initiales. Le rôle joué par l'ambiguïté du cas à juger, sur la nature $\mathrm{du}$ processus d'influence, dans la dynamique $\mathrm{du}$ groupe (normalisation $v s$ polarisation) n'est pas l'objet de cet article. En outre, nous ne sommes pas en mesure de tester cette relation de cause à effet, dans la mesure où nous n'avons pas accès à la description concrète du cas, mais, seulement, au récit que les jurés en font, ce qui cause une distorsion considérable. Néanmoins, nous faisons l'hypothèse qu'un tel lien existe, qui pourrait être l'objet d'une recherche future.

Concernant la troisième variable sur l'impact des médias, les résultats mettent en évidence le réservoir d'ancrage qu'ils constituent pour les jurés. C'est dans celui-ci que ces derniers vont puiser une partie des significations leur permettant de décoder, comprendre et interpréter ce qu'ils sont en train de vivre, cette réalité souvent délicate, complexe et angoissante (sur ce dernier point, voir DurifVarembont, dans ce dossier). Nos résultats vont dans le sens de certains travaux évoquant le rôle des médias dans le rendu de l'information judiciaire (voir Manchec, Somat, Testé, 2004). Toutefois, toutes les «affaires » jugées aux assises ne sont pas soumises à un traitement médiatique égal. Les médias (filmographie, faits divers télévisuels, etc.), en tant que champ de significations, partagé et préexistant, du déroulement du procès, l'institution judiciaire, voire la notion du juste et de l'injuste, permettent, à leur degré, une généralisation fonctionnelle relative à l'expérience vécue, offrent des prototypes et a fortiori des schémas comparatifs d'explication (voir Amsterdam, Bruner, 2000 ; sur l'ancrage, Viaud, 2000).

Notre étude souligne la diversité des sources d'influence, dont les jurés font l'objet et qui viennent, aussi bien des interactions qu'ils entretiennent avec les acteurs du procès (le président, les autres jurés), que des représentations sociales de la justice et de la citoyenneté, relayées par les médias. Ainsi, elle s'inscrit pleinement dans le sens des recommandations de Devine et coll. (2001), à partir des conclusions de leur méta-analyse, effectuée sur environ 200 recherches, menées autour de cette thématique, entre 1955 et 1999. Sans minimiser l'influence des variables légales dans leur jugement, les anciens jurés, interrogés dans le cadre de notre recherche, reconnaissent l'impact de certaines variables extra-légales. La pertinence de ces résultats repose sur la nature et la rareté relative de notre échantillon sans, pour autant, prétendre à une généralisation sur l'ensemble des jurés du système juridique français. Ils nous invitent à prendre en considération l'importance de ces variables dans la construction de «l'intime conviction», pour les recherches futures dans ce champ d'études. 


\section{RÉFÉRENCES}

Amsterdam (Anthony G.), BrunER (Jerome).Minding the law, Cambridge, Harvard university press, 2000.

Beauvallet (Cathy), Cirendini (Olivier).- Cour d'assises : et si demain vous étiez juré ?, Paris, Jalan publications, 2004.

Devine (Dennis J.), Clayton (Laura D.), Dunford (Benjamin B.), SEYING (Rasmy), PRYCE (Jennifer).- Jury decision making. 45 years of empirical research on deliberating groups, Psychology, public policy and law, 7, 2001, p. 622-727.

DoIsE (Willem), Mugny (Gabriel).- Les niveaux d'analyse dans l'influence sociale : la normalisation, dans Mugny (G.), Oberlé (D.), Beauvois (J.-L.), Relations humaines, groupes et influence sociale, Grenoble, Presses universitaires de Grenoble, 1995, p. 239-251.

FINKEL (Norman J.).- Commonsense justice : juror's notions of the law, Cambridge, Harvard university press, 1995.

Guingouain (Gérard), Manchec (Karine), Testé (Benoît)-- Insertion sociale et jugements judiciaires : influences de l'anticipation de délibération en jury en fonction de l'origine ethnique endo ou exo-groupe de l'accusé, Cahiers internationaux de psychologie sociale, 54, 2002, p. 113-124.

JoDELET (Denise).- Place de l'expérience vécue dans les processus de formation des représentations sociales, dans Haas (V.), Les savoirs du quotidien. Transmissions, appropriations, représentations, Rennes, Presses universitaires de Rennes, 2006, p. 235-255.

Kalampalikis (Nikos).- L'apport de la méthode Alceste dans l'étude des représentations sociales, dans Abric (J.-C.), Méthodes d'étude des représentations sociales, Ramonville, Erès, 2003, p. 147-163.

Kalampalikis (Nikos), Moscovici (Serge).- Une approche pragmatique de l'analyse Alceste, Cahiers internationaux de psychologie sociale, 66, 2005, p. 15-24.

Lieberman (Joel D.), SAlES (Bruce D.).- What social science teaches us about the jury instruction process, Psychology, public policy and law, 3, 1997, p. 589-644.

LOMBARD (François).- Les jurés. Justice représentative et représentations de la justice, Paris, L'harmattan, 1993.

Manchec (Karine), Somat (Alain), Testé (Benoît).Justice, équité et démocratie : le rôle de la presse dans les décisions judiciaires. Un point de vue psychosocial, dans Marchand (P.), Psychologie sociale des médias,
Rennes, Presses universitaires de Rennes, 2004, p. 143-165.

Milgram (Stanley).- Soumission à l'autorité, Paris, Calmann-Lévy, 1974.

Moscovici (Serge).- The discovery of group polarization, dans Granberg (D.), Sarup (G.), Social judgements and intergroup relations. Essays in honor of Muzafer Sherif, New York, Springer, 1992, p. 107-127.

Moscovici (Serge), DoIse (Willem).- Dissensions et consensus, Paris, Presses universitaires de France, 1992.

Mugny (Gabriel), Oberlé (Dominique), Beauvois (Jean-Léon).-Relations humaines, groupes et influences sociales, Grenoble, Presses universitaires de Grenoble, 1995.

RAINIS (Natascha).- Les contributions de la psychologie judiciaire et de l'expertise psycho-juridique à l'administration de la justice pénale, dans Le Blanc (A.), Doraï (M.), Roussiau (N.), Bonardi (C.), Psychologie sociale appliquée. Éducation, justice et politique, Paris, In press, 2002, p. 91-116.

REINERT (Max).- Le rôle de la répétition dans la représentation du sens et son approche statistique dans la méthode «Alceste », Semiotica, 147, 1-4, 2003, p. 389-420.

Richard (Ghislaine), Finkelstein (Remy), Oberlé (Dominique), RAINIS (Natascha), ScharnitzKy (Patrick).- Être désigné juré d'assises : réactions et attitudes des citoyens français, Revue européenne de psychologie appliquée, 2008, à paraître.

SCHARNITZKY (Patrick).- Les pièges de la discrimination. Tous acteurs, tous victimes, Paris, Archipel, 2006.

Scharnitzky (Patrick), Kalampalikis (Nikos), Durif-VAREmbont (Jean-Pierre), Mercader (Patricia).Les déterminants psychologiques et sociaux de la fonction de juré d'assises, Rapport de recherche pour la région Rhône-Alpes, Institut de psychologie, Université Lyon 2, 2006.

SCHARNITZKY (Patrick), RAINIS (Natascha).- Juré d'assises : une expérience de la citoyenneté, Le journal des psychologues, 241, 2006, p. 32-36.

SHERIF (Muzafer).- Group influences upon the formation of norms and attitudes, dans Newcomb (T. M.), Hartley (E. L.), Readings in social psychology, Holt, New York, 1947, p. 219-232.

Viaud (Jean).- L'objectivation et la question de l'ancrage dans l'étude des représentations sociales, dans Roussiau (N.), Psychologie sociale, Paris, In press, 2000, p. 89-99. 


\section{ANNEXE}

\section{LE FONCTIONNEMENT DES COURS D’ASSISES}

Les cours d'assises jugent des crimes tels que les attaques à main armée, les agressions, les meurtres, les viols ou encore les actes de pédophilie. Le jury est composé du président de la cour, de ses deux assesseurs et de neuf jurés citoyens. Ceux-ci sont tirés au sort sur les listes électorales. Chaque juré doit avoir la nationalité française, avoir au moins vingt-trois ans, savoir lire et écrire, avoir un casier judiciaire vierge et ne pas travailler dans certains domaines en lien avec la justice ou la police. Il peut alors être tiré au sort et être convoqué pour une session de plusieurs procès se déroulant sur une période donnée dans sa circonscription régionale.

Pour chaque session, une quarantaine de citoyens sont convoqués à une série de procès. Sauf cas exceptionnel, le rôle de juré d'assises entre dans les devoirs du citoyen et nul n'est autorisé à s'y soustraire sous peine d'amende. Lors d'une session, neuf jurés sont à nouveau tirés au sort au début de chaque procès. Cinq d'entre eux peuvent être récusés par l'avocat de la défense et quatre peuvent l'être par le ministère public, uniquement sur la base de leur nom, de leur profession ou de leur apparence physique. Un juré récusé est dispensé de participer au procès et un autre est tiré au sort à sa place jusqu'à ce que le jury populaire soit constitué.

Démarre alors l'audience avec, dans l'ordre, la comparution de l'accusé, la présentation de la liste des témoins, et l'énonciation des faits qui sont reprochés à l'accusé. On procède ensuite aux interrogatoires, puis aux auditions des témoins et des experts.

C'est ensuite la phase des réquisitoires, et des plaidoiries des avocats. Enfin, le président de la cour d'assises donne aux jurés des consignes afin qu'ils se préparent aux délibérations. Lors de ces délibérations, le jury composé des neuf citoyens ainsi que les trois membres de la cour se réunit dans une salle à huis clos pour débattre de l'affaire et pour répondre aux questions posées par le président. La durée de ces délibérations n'est pas fixe. Elle dure aussi longtemps que nécessaire jusqu'à ce que les membres du jury se mettent d'accord à propos des questions qui lui sont posées. Il se prononce sur la culpabilité de l'accusé, sur le degré de sa responsabilité, puis sur l'application de la peine. Une fois le verdict rendu et annoncé, les jurés sont libérés de leur fonction. 